\title{
Mapping of Genes Encoding Coagulation Factors VII and $X$ to the Distal Portion of the $13 q 34$ by Gene Dose Study in a Patient with $\mathrm{r}(13)$
}

To the Editor:

The mapping of genes encoding blood coagulation factors VII (F7) and X (F10) to the 13q34 segment was first suggested by Pfeiffer et al. (1982) on the basis of observations of reduced plasma FVII and FX levels in two patient with a deletion involving the $13 \mathrm{q} 34$ segment. Subsequent investigations on patients with various abnormalities of chromosome 13 have confirmed that monosomy for the $13 \mathrm{q} 34$ segment produced a 50\% decrement (de Grouchy et al., 1984; Gilgenkrantz et al., 1986; Fukushima et al., 1987) while trisomy for the same segment led to a $50 \%$ increment (Gilgenkrantz et al., 1986) in plasma FVII and FX levels. In addition, Scambler and Williamson (1985) have demonstrated on Southern blot analysis of DNA from patients with monosomy for $13 q 34$ using a cloned human F10 gene that the low expression of FX resulted from loss of one copy of the F10 structural genes. Although the loci for F7 and F10 are assumed to form a coagulation gene cluster in this region, localization remains to be detailed at the subband level. In this letter, we describe results of a gene dose study for F7 and F10 in a 3 year 9 month-old female patient with a ring chromosome 13 in which the breakpoint was located at the distal portion of the $13 \mathrm{q} 34$ band.

The patient was born at term to a 30 -year-old mother and an unrelated 32 year-old father. The pregnancy was uneventful, but the delivery was complicated by bleeding due to placenta praevia. The birth weight was $2,264 \mathrm{~g}$. Because of neonatal asphyxia, she was cared for in an incubator for 2 days. Her psychomotor development was slow: head control at 4 months, sitting without support at 1 year 10 months and walking alone at 2 years 7 months. At the age of 3 years 9 months, her body weight was $12 \mathrm{~kg}(-1.7$ S.D. $)$, the height $93.0 \mathrm{~cm}(-1.1 \mathrm{~S} . \mathrm{D}$.) and the head circumference $43.5 \mathrm{~cm}(-3.6$ S.D.). She was found to have microcephaly, long and narrow palpebral fissures, prominent nasal bridge, protruding maxilla, thin lips, anterior displacement of the anus, proximal implantation of the thumbs and bilateral short fifth fingers. Her 2 nd and 4 th toes overlapped onto the $3 \mathrm{rd}$ toes bilaterally. Examinations of the chest and abdomen showed no abnormality. There was no antecedent history of bleeding disorder, and liver function tests yielded normal results.

Conventional cytogenetic study of peripheral blood lymphocytes from the

Received February 15, 1989; revised version received May 28, 1989; Accepted June 2, 1989. 
patient showed a mosaic of many cell lines. The majority of cells examined had monocentric ring chromosome $13(168 / 200,84.0 \%)$, while the minority of cells had monosomy $13(15 / 200,7.5 \%)$, premature chromosome condensation $(4 / 200$, $2.0 \%$ ), dicentric ring chromosome $13(7 / 200,3.5 \%)$, tetracentric ring chromosome $13(2 / 200,1.0 \%)$ and other ring forms $(4 / 200,2.0 \%)$. The presence of a part of the short arm on QFQ-banding and the lack of a nucleolus organizing region on Ag-I staining in the ring chromosome 13 indicated that one of the breakpoints was located on the 13p11.2 (Fig. 1). On the other hand, high-resolution GTG-banding together with RBG-banding showed that most of the $13 \mathrm{q} 34$ segment was retained in the ring chromosome 13 (Fig. 2). On the basis of nomenclature of ISCN (1985), the other breakpoint seemed to be located on 13q3408. The parents had normal karyotypes, and her karyotype was designated as 46,XX,r(13)(p11.2q3408) de novo.

Assays of coagulation factors I, II, V, VII, VIII, IX, X, XI, XII and XIII were performed on the patient and parents. Results showed that in the patient, plasma levels of FVII and FX were $44 \%$ and $48 \%$, respectively, while values of the remaining coagulation factors were normal or even increased (Table 1). All coagulation factors assayed in the parents were within the normal ranges.

The reduction of FVII and FX levels to a half of the normal values, the absence of liver disease and normal plasma levels of the other vitamin $\mathrm{K}$ dependent factors was consistent with hemizygous deficiency of F7 and F10 in the patient. Furthermore, her cytogenetic findings were informative in determining the localization of these coagulation factor genes at the subband level. The combination of various

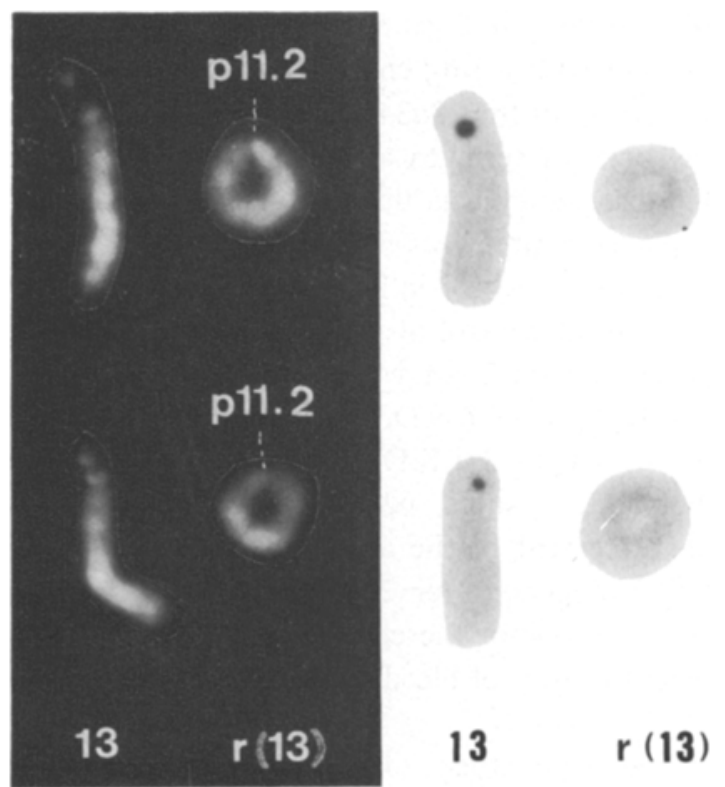

Fig. 1. Partial karyotypes of the patient (left, QFQ-banding; right, Ag-I staining). 

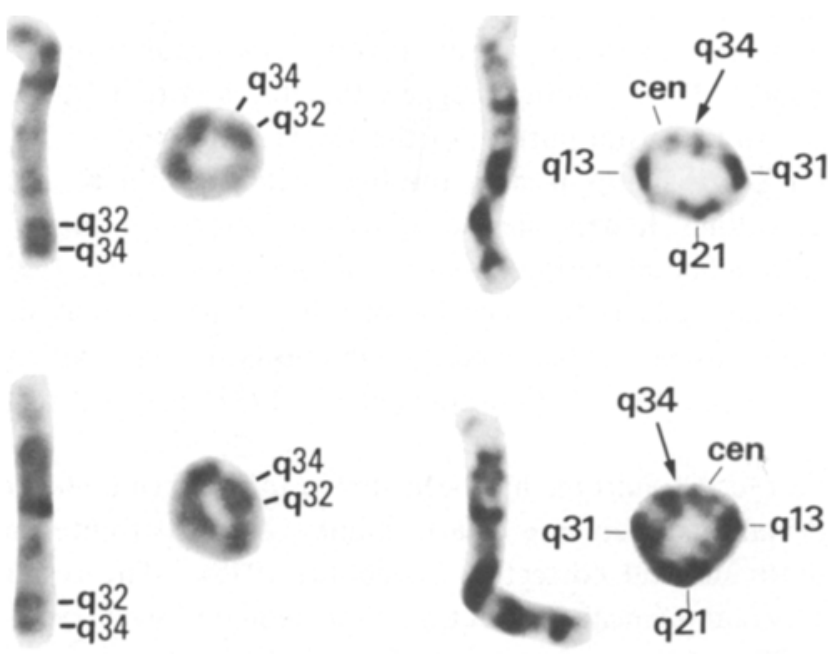

13

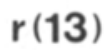

13

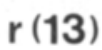

Fig. 2. Partial karyotypes of the patient (left, RBG-banding; right, high-resolution GTGbanding).

Table 1. Results of coagulation factor assays.

\begin{tabular}{ccccc}
\hline & Patient & Mother & Father & Normal range \\
\hline Fibrinogen & $312 \mathrm{mg} / \mathrm{d} 1$ & - & - & $200-400 \mathrm{mg} / \mathrm{dl}$ \\
Factor II & $70 \%$ & - & - & $62-127 \%$ \\
V & $156 \%$ & $110 \%$ & $69 \%$ & $50-150 \%$ \\
VII & $44 \% \mathrm{a}$ & $100 \%$ & $111 \%$ & $65-135 \%$ \\
VIII & $104 \%$ & - & - & $50-150 \%$ \\
IX & $141 \%$ & $109 \%$ & $98 \%$ & $60-140 \%$ \\
X & $48 \% \mathrm{a}$ & $98 \%$ & $122 \%$ & $56-138 \%$ \\
XI & $125 \%$ & - & - & $65-135 \%$ \\
XII & $121 \%$ & $76 \%$ & $123 \%$ & $50-150 \%$ \\
XIII & $131 \%$ & $158 \%$ & $95 \%$ & $72-144 \%$ \\
\hline
\end{tabular}

a Means of two separate measurements.

banding techniques showed that one of the breakpoints in the ring chromosome 13 seemed to have occurred on $13 q 3408$, resulting in monosomy for the distal portion of $13 q 34$ band. There have been three other reported patients with $r(13)$ where a single gene dosage effect for FVII and FX was substantiated (de Grouchy et al., 1984; Gilgenkrantz et al., 1986). Although overt deletion of the long arm 
of chromosome 13 has not been described in any of the 3 patient, it seems likely that ring chromosomes in those patients involved monosomy for the distal portion of the 13q34 band. These findings suggest that the loci of F7 and F10 can be regionally assigned to the distal portion of the $13 q 34$ band.

FVII and FX are synthesized in the liver with vitamin $\mathrm{K}$ as a cofactor. As plasma levels of vitamin $\mathrm{K}$ dependent coagulation factors are known to be markedly reduced from the neonatal period to early infancy (Hathaway, 1987), the interpretation of gene dosage effects for such factors should be cautious during that time. The failure to demonstrate triple gene dosage effects in some patients with trisomy 13 (de Grouchy et al., 1982; Fukushima et al., 1987) may be related to this age factor.

Finally, the $13 q$ - syndrome has been shown to be often associated with various internal malformations involving heart, kidney and gastrointestinal tract which require immediate surgical correction (Niebuhr, 1977). The results of our study provide an important clinical implication that neonates with the $13 q-$ syndrome may be at high risk of having excessive bleeding due to the deficiency of FVII and FX after a major operative procedure.

\section{REFERENCES}

Fukushima, Y., Kuroki, Y. and Lizuka, A. 1987. Activity and antigen of coagulation factors VII and $\mathrm{X}$ in five patients with abnormal chromosome 13. Jpn. J. Human Genet. 39: 91-96.

Gilgenkrantz, S., Briquel, M.-E., Andre, E., Alexandre, P., Jalbert, P., Lemarec, B., Pouzol, P. and Pommereuil, M. 1986. Structural genes of coagulation factors VIl and $X$ located on 13q34. Ann. Génét. 29:32-35.

de Grouchy, J., Dautzenberg, M.-D., Turleau, C., Beguin, S. and Chavin-Colin, F. 1984. Regional mapping of clotting factors VII and X to 13q34. Expression of factor VII through chromosome 8. Hum. Genet. 66: 230-233.

Hathaway, W.E. 1987. Hemostasis. In Pediatrics, Rudolph, A.M., ed., 18th Ed., Appleton and Lange, Norwalk, pp. 1075-1076.

ISCN. 1985. Specification of breakpoint. In An International System for Human Cytogenetic Nomenclature. Harnden, D.G. and Klinger, H.P., eds., Karger, Basel, pp. 28.

Niebuhr, E. 1977. Partial trisomies and deletions of chromosome 13. In New Chromosomal Syndromes, Yunnis, J.J., ed., Academic Press, New York, pp. 273-292.

Pfeiffer, R.A., Ott, R., Gilgenkrantz, S. and Alexandre, P. 1982. Deficiency of coagulation factors VII and $X$ associated with deletion of a chromosome 13 (q34). Evidence from two cases with 46,XY,t(13;Y)(q34;q11). Hum. Genet. 62: 358-360.

Scambler, P.J. and Williamson, R. 1985. The structural gene for coagulation factor $X$ is located on chromosome 13q34. Cytogenet. Cell Genet. 39: 231-233.

Ryozo Kasai, ${ }^{1}$ Kouji Narahara, ${ }^{2}$ Hiroshi Namba, ${ }^{2}$ Kazushiro TsujI, ${ }^{2}$ Tsunenori Matsubara, ${ }^{2}$ Kei Hiramoto, ${ }^{2}$

Yuji YoKoYAma, ${ }^{2}$ and Hiroshi Kimoto ${ }^{2}$

'Asahigawa Jidoin Hospital for Handicapped Children, Okayama 703, Japan

${ }^{2}$ Department of Pediatrics, Okayama University School of Medicine, Okayama 700, Japan 